\title{
Embarazo en la adolescencia: experiencia de la ASCP
}

\author{
Patricia Sánchez Jaramillo, Psc.*; Néstor Mendieta Cruz, MD**
}

RESUMEN OBJETIVO: Sistematizar la información contenida en historias clínicas de adolescentes embarazadas atendidas por ASCP, para presentar un perfil médico-psico-social, determinar la incidencia y tipo de complicaciones obstétricas y clasificar los antecedentes ginecobstétricos y psicosociales de esta población.

MATERIAL Y METODOS: Se realizó un estudio descriptivo de tipo retrospectivo sobre 555 historias clínicas. Se relacionó la edad de las pacientes con sus antecedentes psicosociales y ginecobstétricos y con la patología perinatal.

RESULTADOS: Se presentó tendencia a reproducir los antecedentes familiares en cuanto a madresolterismo y embarazo en la adolescencia; no se presentó promiscuidad y el promedio de compañeros sexuales fue de 1.3. Muy pocas conocían o usaban métodos anticonceptivos. Se encontró un índice importante de embarazo previo. Aunque la incidencia de patología durante el embarazo fue alta, ésta fue de muy baja complejidad. No se encontró un índice significativamente mayor al de la población general en cuanto a complicaciones durante el parto y el puerperio o en el recién nacido.

CONCLUSIONES: Las investigaciones e intervenciones no se deben circunscribir a los aspectos médicos del embarazo en la adolescencia. Es necesario realizar una educación sexual amplia y veraz. Se proponen algunos criterios para la intervención en el embarazo en la adolescencia.

PALABRAS CLAVES: Adolescencia, embarazo, riesgos ginecobstétricos, antecedentes psicosociales.

SUMMARY OBJECTIVE: To sistematize the information conteined in the clinic records of 555 pregnant adolescents attended by ASCP, in order to obtain a medical-psycho-social profile to determine the influence and the kind of obstetrics and psychological antecedents of this population.

MATERIAL AND METHODS: The ASCP completed descriptive research based on about 555 clinic records. In this research is given a description of psycho-social and gyneco-obstetrics antecedents, and the pregnancy pathology by age of group.

RESULTS: In this study it was observed that the pattern of pregnancy and the status of single mothers tended to reproduce the pattern of their families. The stady dis not find promiscuitty. The average number of sexual pathners was 1.3 . Not many teenagers had known an used contraceptive methods. The research found a important previous pregnancy rate. Althogh incidence of pathology during pregnancy was high, it was not very complex. The rate of complications during delivery and after birth was not significantly greater than that of the general population.

CONCLUSIONS: Research must not be limit to the medical aspects of adolescence pregnancy. It is necesary to offer a sexual education more truthful and with wider scope. The study proposed criteria for the intervention in the pregnancy of adolescents.

KEY WORDS: Adolescence, pregnancy, gineco-obstetrics risks, psycho-social antecedents.

\section{Introducción}

En la actualidad el embarazo en la adolescencia se perfila como una de las problemáticas más sentidas por las sociedades latinoamericanas, toda vez que se aprecia y se prevé un aumento sostenido en su incidencia, frente al cual no se ha desarrollado mayor conocimiento y por lo tanto, tampoco se dispone de estrategias de intervención suficientes para prevenirlo y atenderlo.

En efecto, estadísticas colombianas proporcionadas por Minsalud, muestran al embarazo y a los factores

* Psicóloga, Directora Asociación Salud con Prevención

** Coordinador Area de Investigación. Asociación Salud con Prevención. relacionados con él, ocupando la primera causa de consulta externa, las cinco primeras causas de egreso hospitalario y seis de las ocho primeras causas del mismo en la población femenina entre los 15 y 19 años, así como la segunda causa de egreso hospitalario en mujeres entre 10 y 14 años, lo cual manifiesta claramente la gravedad de la problemática referida.

Así mismo, las estrategias para prevenir esta problemática no parecen arrojar resultados halagüeños; en Colombia por ejemplo, según el EPDS (1) tan sólo el $5.6 \%$ de las mujeres entre los 15 y 19 años usa algún método anticonceptivo y sólo el $36.9 \%$ de aquellas que dentro del mismo rango de edad se encuentran unidas, recurre al uso de dichos métodos. En 1990 el EPDS 
también afirmaba que 1'780.000 jóvenes entre los 15 y 19 años ya eran madres o tenían un embarazo en curso, presentándose para esta población una tasa de fecundidad del 70 por mil. Cabe anotar el insignificante descenso de dichas tasas durante los últimos veinte años, tanto en la población descrita como en la población general. De esta forma, según el DANE, en 1987 el $7.5 \%$ de los nacimientos en el país eran producto de embarazos en la adolescencia (2).

De otro lado, si tomamos en cuenta que a nivel mundial el incremento absoluto de los adolescentes entre los 10 y 19 años será del $27 \%$ en los próximos 35 años, que el $86 \%$ del total corresponderá a los países en vía de desarrollo y que la vida sexual de los adolescentes es cada vez más activa y precoz, bien puede asegurarse que nos encontramos ante un fenómeno social altamente complicado y peligroso que plantea un difícil reto a las diversas disciplinas comprometidas en su resolución y control (3-4).

Esta situación ha propiciado recientemente la realización de investigaciones tendientes a estudiar sus causas y posibles soluciones, siendo el propósito general de la mayoría de trabajos, empezar a definir "quién es la adolescente que se embaraza" (5). Paradójicamente, esta urgencia es la producción del conocimiento pertinente, debe implicar un mayor cuidado metodológico, de manera que haya un respeto riguroso para la selección de muestras sin sesgos y para el uso de la estadística adecua$\mathrm{da}$, teniendo en cuenta que se trata de un "tema que realmente está en su infancia como área de investigación" (6).

Por todas estas razones, la ASCP luego de haber trabajado ininterrumpidamente durante 5 años con adolescentes embarazadas, acompañándolas a través de un control prenatal y de una asesoría psicológica, consideró necesario y oportuno realizar la sistematización de esta información y el análisis estadístico de la misma. El registro de los datos de las adolescentes atendidas, junto con su análisis y discusión, puede aportar en la identificación de las características médicas y psicosociales de la adolescente embarazada y consecuentemente en la construcción de estrategias, metodologías y programas de intervención que permitan enfrentar con éxito una problemática que a todos preocupa.

\section{Propósitos}

1. Fundamentar a través del análisis de una experiencia, un programa de intervención para la adolescente embarazada.

2. Dar a conocer los resultados de la experiencia, que por cinco años ha desarrollado la ASCP en el manejo del embarazo de la adolescente.

3. Proponer nuevos campos de trabajo en la problemática del embarazo en la adolescencia.

\section{Objetivos}

Realizar un perfil médico-psicosocial de las usuarias atendidas en el servicio de la asociación salud con Prevención.
Sistematizar la información recogida a través de la Historia Clínica de la ASCP, sobre el embarazo en la adolescencia y hacer su análisis estadístico.

\section{Metodología}

La población de usuarias de los servicios de la ASCP, pertenece a las clases baja y media baja que se ubican en algunos barrios populares del norte de Bogotá y que acuden a los centros de salud y Centros de Atención Médica Inmediata del Servicio Distrital de Salud. La edad del grupo oscila entre los 14 y 19 años.

De aproximadamente 1800 adolescentes atendidas, se seleccionaron 555 historias clínicas que contenían la información requerida para el estudio. Con el fin de interpretar la información referida a la patología perinatal, se diseñó un formulario de sistematización de datos, el cual fue aplicado a cada una de las historias clínicas seleccionadas.

Los datos obtenidos fueron analizados a través de una distribución de frecuencias, expresada en promedios y porcentajes y llevados a incidencias por edades para facilitar su confrontación e interpretación.

\section{Resultados}

La distribución por edades fue la siguiente: 14 años $3.9 \%$; 15 años $-7.7 \% ; 16$ años $-19.2 \%$; 17 años $-23.2 \%$; 18 años $-25.1 \%$ y 19 años $-25 \%$.

\section{Características psicosociales}

En cuanto a la escolaridad, el $62 \%$ de las usuarias tenían algún grado de secundaria, el $36 \%$ alcanzaba sólo estudios primarios y el $2 \%$ superiores.

La mayoría se dedicaban al hogar (60\%) y tan sólo un $16 \%$ se encontraban laborando.

Un $40 \%$ del grupo pertenece a familias medianas (56 miembros), un $28 \%$ a pequeñas (menos de 4 miembros) y un $28 \%$ a familias con más de 6 integrantes.

El 74\% de las adolescentes tienen antecedentes familiares de madresolterismo y/o embarazo en la adolescencia. Se aprecia también una relativa estabilidad en las familias de origen (bajo índice de separaciones y divorcio) pero una alta incidencia de maltrato en el hogar $(20 \%)$. (Ver gráfico 1$)$.

En cuanto a la vida sexual de la adolescente, ésta se inicia en promedio hacia los 15.5 años y el promedio de compañeros sexuales que han tenido en su vida es de 1.3. Así mismo, el $46 \%$ del grupo se tomó 6 meses o más para iniciar relaciones sexuales con su pareja y sólo el $24 \%$ lo hizo antes de los 6 meses. De esta manera el embarazo fue producto del noviazgo en un $76 \%$ de los casos; del matrimonio en un $26 \%$; de la amistad en un $2 \%$ y de violación en un $2 \%$.

Por otra parte, el $54 \%$ afirma haber recibido algún tipo de educación sexual, básicamente a nivel escolar, pero tan sólo el $28 \%$ conoce los métodos anticonceptivos. Así mismo, la mayoría manifestaron que esta educación sexual hacía referencia exclusivamente a los aspectos anatómicos y fisiológicos. En el mismo orden de ideas, 
del grupo que conocía los métodos anticonceptivos sólo la mitad (14\% de la muestra total) usaban algún método, de preferencia de tipo natural. (Ver gráfico 2).

De otro lado, al momento de la entrevista (realizada durante el embarazo) la mayor parte del grupo $(67 \%)$ se encontraba casado (11\%) o en unión libre (56\%). De este grupo a su vez, el $54 \%$ convivían con sus familias de origen, sobre todo con la familia del compañero.

En cuanto a los compañeros de las adolescentes, la mayoría tienen 5 o más años que ellas; es decir, no son coetáneos y un $88 \%$ se encuentra empleado aunque sea en el sector informal de la economía. La escolaridad del $64 \%$ de ellos incluyó algunos años de secundaria y un $6 \%$ tuvo estudios superiores.

\section{PERFIL PSICO-SOCIAL. ANTECEDENTES FAMILIARES}

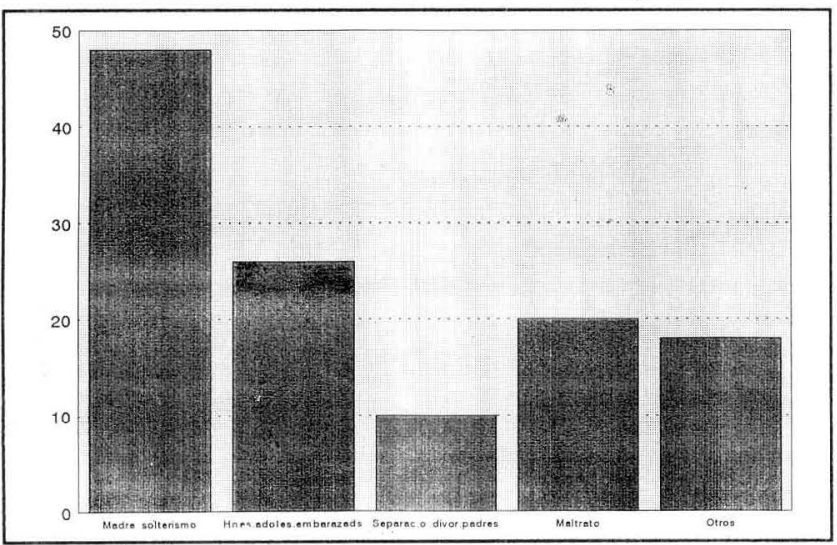

Gráfico 2

EDUCACION SEXUAL

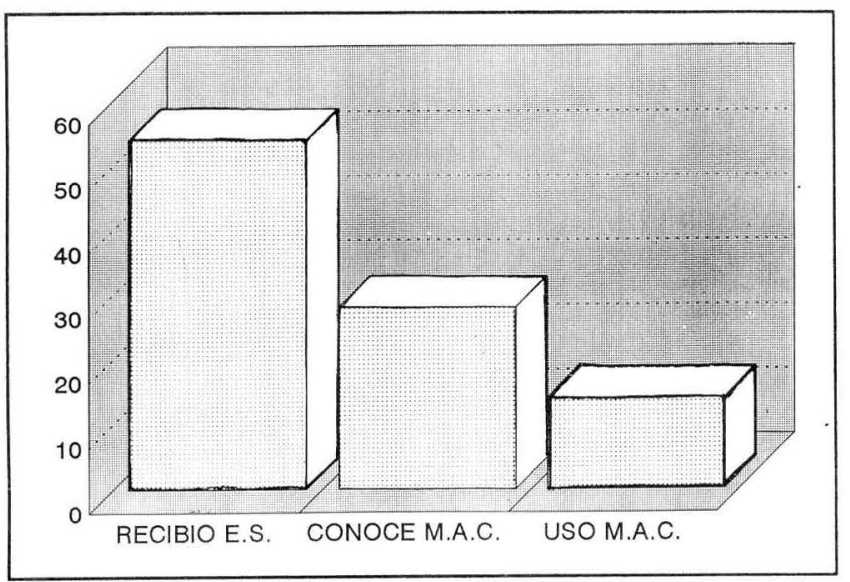

\section{Antecedentes ginecobstétricos}

Se pudo apreciar cómo el promedio de menarquia fue progresivamente más precoz a menor edad de la paciente, lo cual puede significar que cada día la edad en que la mujer se hace fértil es más temprana.
Un $18.5 \%$ del grupo refirió ciclos menstruales irregulares. También se debe resaltar que el $12.6 \%$ de nuestras usuarias ya había tenido al menos un embarazo con anterioridad al que cursaba en el momento de su consulta. En el grupo de 19 años, una de cada 5 pacientes presentaba este antecedente. La incidencia de abortos anteriores fue de $4.5 \%$. (ver gráfico 3 ).

\section{Gráfico 3 \\ INCIDENCIA EMBARAZO PREVIO SEGUN EDAD}

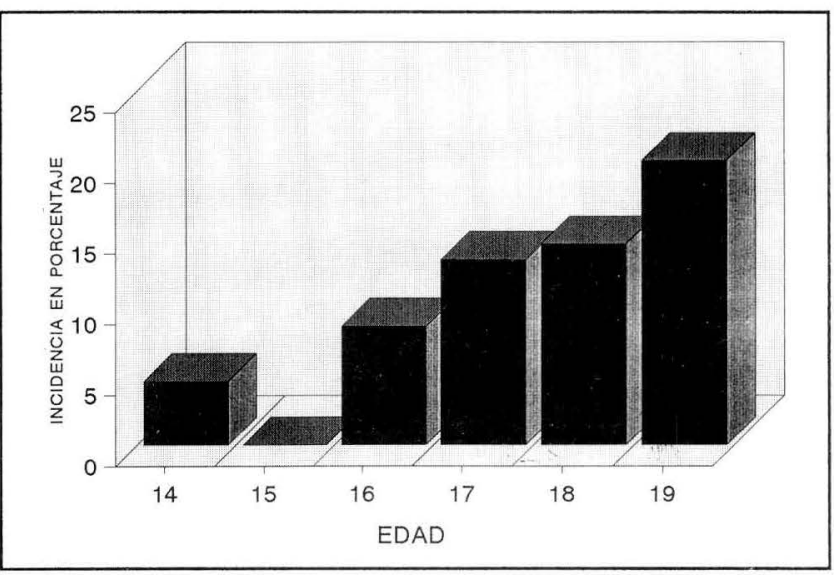

\section{Evolución de los embarazos}

El promedio de controles prenatales para el grupo fue de 4.58 .

El estudio señala que la incidencia de patología durante el embarazo fue de $46.1 \%$, siendo mayor en el grupo de 14 años $(54.5 \%)$ y decreciendo progresivamente hasta los 19 años en donde fue de un $40.3 \%$. No obstante, el tipo de patología fue de muy baja complejidad, correspondiendo un $61.7 \%$ a leucorreas, $21.5 \%$ a bacteriurias e infecciones urinarias y un $11.5 \%$ a anemia y ferropenia. La incidencia de pre-eclampsia fue de sólo $0.9 \%$.

En cuanto al parto, las complicaciones fueron relativamente pocas $(14.5 \%)$ al igual que el índice de partos inferiores a las 38 semanas $(16.1 \%)$. La incidencia de cesáreas fue de un $7.8 \%$. Cabe anotar que la relación menor edad-mayor incidencia de complicaciones, fue mucho menos significativa frente al parto que frente a la patología del embarazo, lo cual podría deberse al manejo efectuado en los controles prenatales.

En lo que hace referencia a los recién nacidos, el $8.2 \%$ presentaron complicaciones y la incidencia de bajo peso al nacer fue de $7.05 \%$, similar a la descrita en otros estudios (7). La incidencia de mortalidad fue de $0.8 \%$, a este respecto Duarte Contreras refiere unas incidencias que alcanzan el 5 ó el $6 \%$ (8). El 1.2\% presentaron malformaciones congénitas, índice equivalente con el de la población general.

Finalmente, las complicaciones durante el puerperio fueron del $2.4 \%$, siendo la más frecuente la endometritis (1.6\% del gran total). (Ver gráficos 4, 5, 6 y Tabla 1). 
Gráfico 4

ADOLESCENTES QUE PRESENTARON PATOLOGIA EN EL EMBARAZO: INCIDENCIA POR EDADES

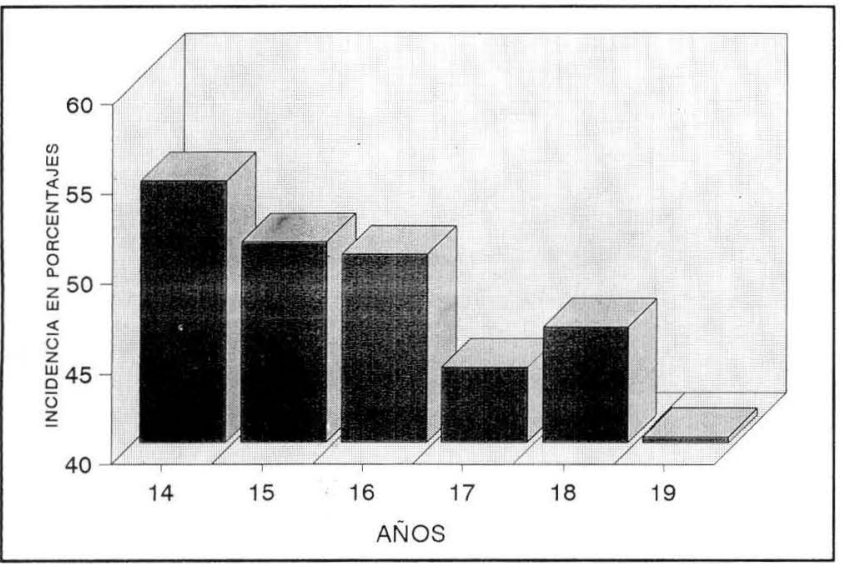

Gráfico 5

COMPLICACIONES EN PARTO. INCIDENCIA POR EDADES

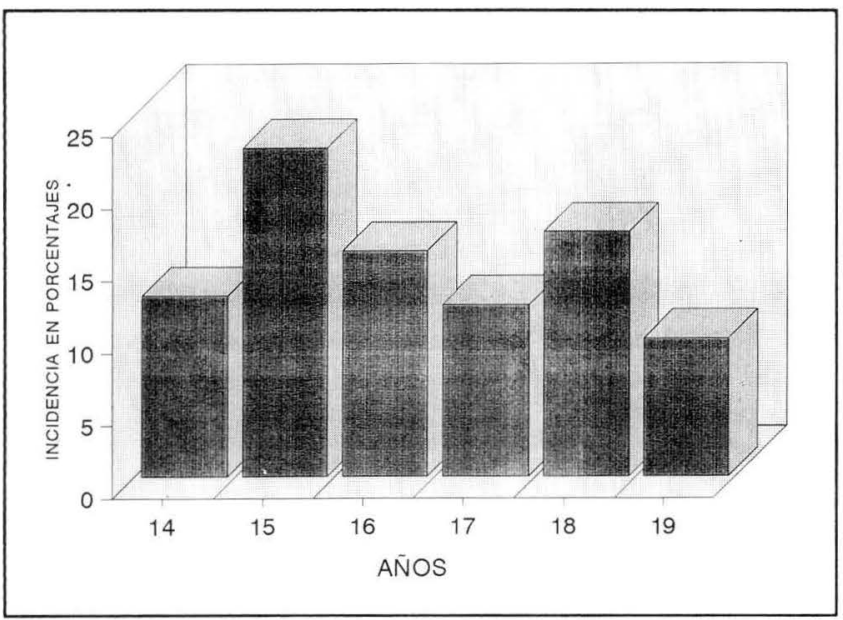

Gráfico 6

INCIDENCIA DE BAJO PESO AL NACER SEGUN EDAD DE LA MADRE

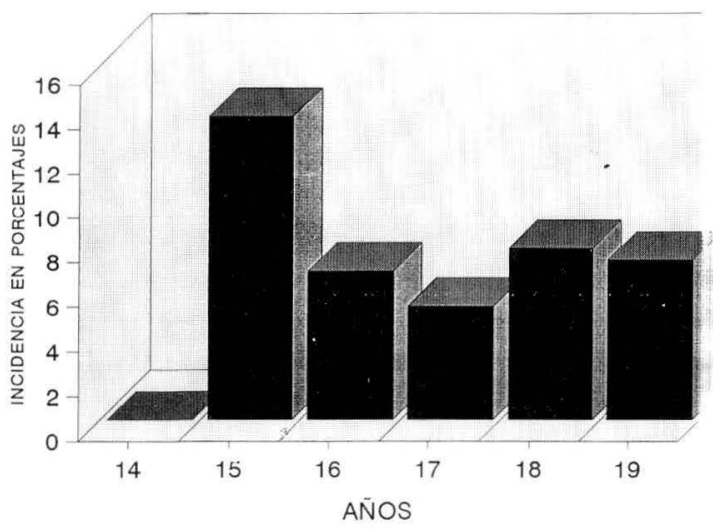

Gráfico 7

COMPLICACIONES EN LOS RECIEN NACIDOS. INCIDENCIA SEGUN EDAD DE LA MADRE

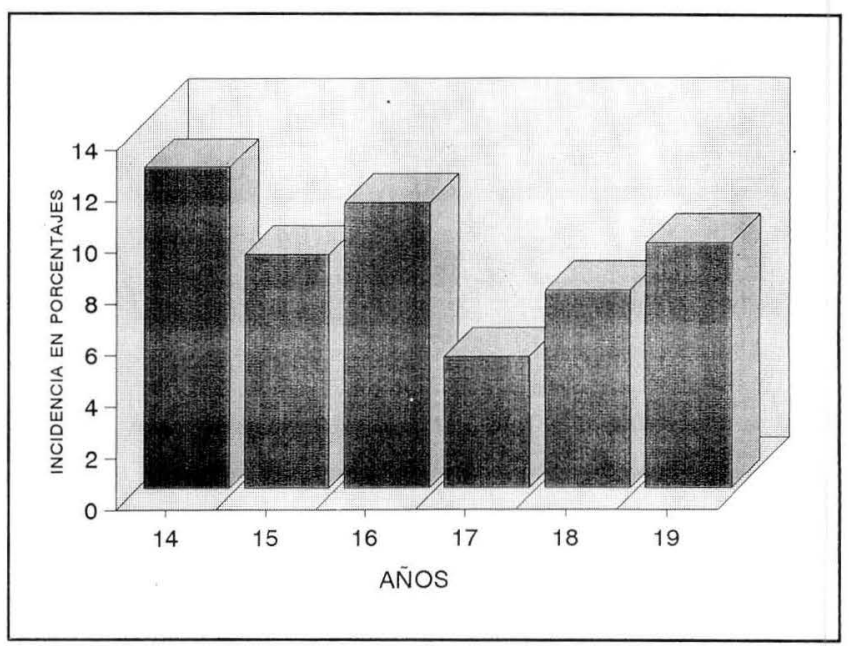

Tabla 1

\section{EMBARAZO EN LA ADOLESCENCIA INCIDENCIAS}

\begin{tabular}{|lr|}
\hline . Patología en el embarazo & $46.1 \%$ \\
\hline · Mortalidad perinatal & $0.8 \%$ \\
\hline . Complicaciones en el parto & $14.5 \%$ \\
\hline . Complic. en el recién nacido & $8.2 \%$ \\
\hline Complic. en el puerperio & $2.4 \%$ \\
\hline . Bajo peso al nacer & $7.05 \%$ \\
\hline . Malformaciones & $1.2 \%$ \\
\hline . Cesárea & $7.8 \%$ \\
\hline
\end{tabular}

\section{Discusión}

Muchos de los resultados obtenidos en este estudio no correlacionaron con los referidos por la literatura. La incidencia de patologías graves por ejemplo, fue muy inferior a la referida, al igual que la mortalidad perinatal. Así mismo, las incidencias de complicación en el recién nacido y de parto pretérmino fueron también inferiores a las que presentan otros autores (9-10).

Pensamos que esto puede deberse al hecho de que la mayor parte de estudios acerca del embarazo en la adolescencia en nuestro país, han sido realizadas con la población que consulta hospitales universitarios los cuales, de acuerdo con las normas del sistema nacional de salud, deben atender patologías de alta complejidad (1112).

No obstante, es importante recalcar la gran contradicción que arroja los diferentes estudios realizados. Algunos de ellos señalan al embarazo en la adolescencia como una entidad de muy alto riesgo (13-14), otros responsabilizan de dicho riesgo a la alta incidencia de 
toxemia (Duarte Contreras señala un 11.5\%) (15), hallazgo que no se corresponde con el estudio actual. Finalmente, otros afirman que el riesgo obstétrico del embarazo en la adolescencia es superable con un adecuado control prenatal (16-17).

De otro lado, resulta alarmante el índice de embarazo previo en este grupo, lo cual nos muestra que se trata de una población con bastantes probabilidades de reincidencia.

Se corroboran muchas afirmaciones de la literatura con respecto al perfil psicosocial de la adolescente que se embaraza; por ejemplo, su tendencia a reproducir la historia familiar generación tras generación, el predominio de uniones consensuales y el hecho de que la mayor parte de los compañeros de las usuarias sean bastante mayores que ellas (18-19).

Sin embargo, la adolescente que consultó nuestros servicios contaba con algún tipo de apoyo por parte del compañero y de la familia durante su embarazo, aunque es importante advertir que no tenemos datos sobre la persistencia de este apoyo a más largo plazo. A este respecto nuestra experiencia nos conduce a suponer que se hace considerablemente menor. No obstante, recalcamos que la adolescente que recibimos no es un ser desprotegido condenado a la indigencia y a la prostitución, sino que es una persona con recursos que pueden ser potenciados a través de intervenciones apropiadas (20).

La información obtenida con respecto a la vida sexual de la adolescente manifiesta que no existen en esta población ni promiscuidad ni una gran precipitud para iniciar relaciones sexuales, como muchas veces se plantea (21-22).
También se deben resaltar las enormes deficiencias en la educación sexual que este grupo recibió, manifestándose claramente que la planificación familiar y el uso de anticonceptivos exige mucho más que la simple información sobre los mismos.

\section{Conclusiones}

1. Es necesario realizar más investigaciones sobre este tópico que no se circunscriban a los aspectos médi$\cos$ y que realicen un seguimiento a estas adolescentes de manera que conozcamos las consecuencias que a largo plazo tiene el embarazo en la adolescencia tanto a nivel médico como psicosocial.

2. Nuevamente se evidencia la necesidad de una educación sexual que no esté enfocada en la anatomía y la fisiología y que no sea distorsionada por prejuicios que conviertan el embarazo en la adolescencia en una enfermedad con frecuentes consecuencias mortales.

3. Finalmente, pensamos que la atención del embarazo en la adolescencia amerita un trabajo interdisciplinario que debe apoyarse en algunos criterios esenciales como la empatía, el consenso y la autogestión. Es decir, debe tener elementos que permitan la construcción de una relación con la paciente que contemple su situación, frecuentemente de frustración y vergüenza, pero que también promueva el desarrollo de los recursos de toda índole que la adolescente posee para la asimilación positiva de su nueva condición de vida.

\section{BIBLIOGRAFIA}

1. Encuesta de Demografía y Salud de PROFAMILIA. 1990.

2. DANE. Colombia Estadística. Volumen 1, Nacional. 1990.

3. Klein R. "Programas de intervención para adolescentes" en: "La psicología en el ámbito perinatal" INPER. Méjico. 1988; 488.

4. Reyista SEMANA. Abril 16 de 1991. Pág. 72.

5. Tolbert K. "La adolescente embarazada: características y riesgos psicosociales”. En la Psicología en el ámbito perinatal. INPE. Méjico.

6. Ibídem. Pág. 42.

7. Duarte A. "Ginecología de la niña y de la adolescente". Bogotá. Editorial Presencia. 1988; 451.

8. Ibídem. Pág. 452.

9. Martínez E. "Análisis de la incidencia de recién nacidos de bajo peso en adolescentes embarazadas". Rev. Chil. Obstet. Ginecol. 1987; (1) 52 .

10. Cruz Y. Rev. Cuba Obstet. Ginecol. 1989; (3)15.

11. Pardo F., Uriza G. "Estudio de embarazo en adolescentes en 11 instituciones colombianas", en: Revista Colombiana de Obstetricia y Ginecología. 1991; 42(2): 135

12. Encizar R. "Embarazo en adolescentes - Instituto Materno Infantil (1980-1985)". Bogotá 1987.

13. Rebolledo A., Atalah E. "Riesgos nutricionales en embarazadas adolescentes" en: Rev. Chil. Nutr. 1986; 14(3): 320
14. Romo JG. "Embarazo, parto y puerperio en primigestantes precoces; Hospital de Salamanca (1978-1982)". Boletín Hosp. San Juan de Dios $1985 ; 32(2): 88$

15. Duarte A. "El embarazo en la adolescencia es siempre de alto riesgo?" En: Rev. Col. Obstet. y Ginec. 1975.

16. Arechavaleta H., Uzcátegui O. "Embarazo en adolescentes". Rev Obstet. Ginecol. Venezuela 1985; 45(2): 38

17. Pérez J., Torres A. "Repercusión del embarazo en la salud perinatal de la adolescente". En: La psicología en el ámbito perinatal. INPER, Méjico 1986; 386.

18. Fernández A. y otros. "Adolescentes embarazadas: relación de pareja y apoyos familiares" En: La psicología social en Méjico. Asociación Mejicana de Psicología Social. 1988; 2.

19. Basualto 1987; Casarotto 1985 y otros citados por Erika K. en "Aspectos psicosociales de la embarazada y madre adolescente" en: Compromisos antes de tiempo. CORSAPS 1988; 55.

20. Bustos 1985; Valenzuela 1985. Citado por: Erika K. Op. Cit. 1988; 53.

21. Ibídem.

22. Tolbert K. Op. Cit. 418. 